\title{
Survey on Classifiers: Implemented for Fracture Detection
}

\author{
Jagruti S. Bhalke \\ Department of E \& Tc \\ Vishwakarma Institute of \\ Technology \\ City- Pune, Maharashtra. \\ Country- India
}

\author{
M. V. Wyawahare \\ Department of E \& Tc \\ Vishwakarma Institute of \\ Technology \\ City- Pune, Maharashtra. \\ Country- India
}

\begin{abstract}
This paper deals with classifiers that have been used for bone fracture detection in the past few years. The authors have made attempts to survey papers from different modalities. This guided us to study different classifiers that have been applied to images obtained from X-Ray. The study presented in simple manner so that it will be easy to understand and will help reader to study classifiers.
\end{abstract}

\section{General Terms}

Medical imaging, machine vision, biomedical image analysis

\section{Keywords}

Survey, bone fracture, classifiers

\section{INTRODUCTION}

Medical imaging is a field that provides 'Quality healthcare' for the patients by using various automated techniques and procedures. Medical imaging has changed the face of clinical medicine. There is a growing interest during the last decades in finding diagnostic methods for skeletal system diseases. Among these diseases, fractures detection and treatment, which affects people of all ages, is growing importance in modern society. Until recently, X-Ray images were maintained as hard film copy. [1]

Now-a-days, X-Ray machines produce extremely high-quality images for radiologists to interpret. X-Ray image classification is an area that has attracted researchers for the past few decades. [1] X-Ray is one the oldest and frequently used devices, as they are non-invasive, painless and economical. [2]

A bone $\mathrm{x}$-ray makes images of any bone in the body, including the hand, wrist, arm, elbow, shoulder, foot, ankle, leg (shin), knee, thigh, hip, pelvis or spine. A typical bone ailment is the fracture, which occurs when bone cannot withstand outside force like direct blows, twisting injuries and falls. [2]

Development in machine vision can enable doctors to use computers as second opinion to diagnose fractures in bone. [3]. Such systems called Computer aided diagnosis (CAD) systems can prove very useful to analyze large volumes of medical data, as well as improve the accuracy of interpretation while reducing time for diagnosis.

In medical applications, sensitivity in detecting medical problems and accuracy of the detection (also called specificity) are two important performance measures that are often in conflict. [4]

\section{RELATED WORK}

There are various fracture detection techniques, according to one of survey done by [3] they have listed 7 methods including its approach towards problem solving. Using one of these methods followed by proper classifier one can solve the problem of fracture detection.

\subsection{X- Ray}

\subsubsection{Calculating bone alignment}

[5] Y Jia and Y Jiang present a method that outlines fractured bones in an X-ray image of a patient's arm within casting materials, and displays the alignment between the fractured bones. A geodesic active contour model with global constraints is applied to segment the bone region. A prior shape is collected and used as a global constraint of our model. A maximum-likelihood function is derived to provide feedback for each evolving process. Experimental results show that the method produces the outlines of the fractured bones on the low contrast X-ray images robustly and accurately. [3]

\subsubsection{Mathematical morphology}

[6] JIAN LIANG et al. have proposed morphological method to identify fractures in tibia bones. Before segmentation, the original image is dynamically divided into several intervals to help find out the smallest interval with the target. The small regions are then automatically threshold using Otsu method .To promotes the accuracy of segmentation and to avoid over or under segmentation; the segmentation result obtained is examined using statistical method. Depending on the test results the segmented image is adjusted.After the second segmentation, the steps of verification and adjustment are required to repeat till the test result conforms to any one of the stopping conditions. When the segmentation is finished, the target image will no long have tough areas.

This is followed by mathematical morphology to extract the target border and cover the boundary of fractures. Then by superposing the target border image and covering the extracted skeleton, the precise location of fractures can be recognized. [3]

\subsubsection{Gradient analysis}

[7] Martin Donnelley et al. developed a method of automatically detecting fractures in long bones. First the edges are extracted from the x-ray image using a non-linear anisotropic diffusion method (the affine morphological scale space) that smoothes the image without losing critical information about the boundary locations within the image. Then a modified Hough transform with automatic peak 
detection is used to determine parameters for the straight lines that best approximate the edges of the long bones. The parameters used to approximate the long bone edges are then used for centreline approximation, diaphysis segmentation and fracture detection in the segmented region. [3]

\subsubsection{Using vertical integral projection}

[8] ZHENG Wei et al. deal with the problem of automatic interpretation of fracture injury site in femur bones by converting it to bone shape identification problem. Depending on the shape of the bone segment, the fractures locations can be identified as - the proximal, middle or the distal part of femur bone. The algorithm conducts vertically integral projection for each pre-processed bone region in X-ray image, and combines the projection curves. After that, the Muller AO coding standing for the fracture injure site is judged based on analysis of subsection variances of curves. If the variance ratio is greater than a fixed value, the program output a numeric code " 1 ", if the variance ratio is less than a fixed value, the program output a numeric code " 3 ". Otherwise, the program output a numeric code " 2 ". Then the fracture injury site is automatically interpreted according to the code matching rules. [3]

\subsubsection{Using discrete wavelet transforms}

[9] Rebecca Smith et al. present a fracture detection method for the pelvic ring based on Discrete Wavelet Transform. DWT is applied to windows extracted from the extracted from the ring as defined by prior automated region segmentation. The chosen wavelet coefficient is used to reconstruct an image that highlights the bone boundary. This is followed by morphological operations on its binary image. The bone boundaries of the ring are then traced using the 8neighborhood of each edge pixel and returned as a matrix of pixel positions. If there is no fracture, the window will contain a single uninterrupted boundary; otherwise there will be multiple boundaries depending on the types and number of fractures. [3]

\subsubsection{By minimization of fuzzy index measure}

[10] The paper introduces a procedure for crack detection in $\mathrm{X}$-ray image, which is based on the minimization of a fuzzy measure. The image histogram is divided into three fuzzy subsets using iterative approach to obtain subsets parameters. The obtained parameters were used as initial estimates and each pixel in the fuzzy regions were classified as belonging to one of the sub-sets by minimizing the fuzzy index. After segmenting the image into three regions, the background and skin regions are removed to detect the cracks in the bone region. A binary image thus obtained contains cavities or holes. A hole-filling step utilizing the morphological operation is then applied to the binary image to fill these spots and create a temporary image. The temporary image is subtracted by the original binary image to isolate the small pots. Morphological filtering functions (erosion followed by dilation) are then used to screen noise or undesirable spots using the iteration number as an operational parameter. The morphological operation can eliminate or maintain the spots on the image according to their area size. This is done to identify possible infestation sites among small spots segmented from the binary image. The output of this process is a binary image containing the crack. [3]

\section{CLASSIFIERS}

This section has been organized based on classifiers used in fracture detection field. This will help the reader in understanding the details about the classifiers.
According to survey paper there are basic standard classifiers namely BPNN, ANN, Bayesian, Gini-SVM, Probabilistic SVM, SVM, Hierarchy of SVM, NB, Binary / Linear Classifiers. Combining these one or two classifiers Hybrid classifiers will be obtained.

\subsection{Standard Classifiers}

\subsubsection{Feed Forward Back Propagation Network (BPNN)}

[1] Mahendran Presents ensemble system for the purpose of detecting fractures in X-ray images. Total 11 texture features used are GLCM features namely Contrast, Homogeneity, Energy, Entropy, Mean, Variance, Standard Deviation. Apart from this Gabor Orientation (GO), Markov Random Field (MRF), and Intensity Gradient Direction (IGD) features. Hold-out method is used for Partitioning dataset and Majority voting scheme is used for Aggregation of obtained results.

BPNN with the tangent-sigmoid transfer function is considered. The weights and biases of the neural networks are initialized randomly, and the number of neurons in the hidden node is determined heuristically as inputs + outputs. This is followed with the results proving that instead of single classifier fusion classifier gives better accuracy.

\subsubsection{Bayesian}

[11] Lim, Sher Ee, et al describes an approach in detecting fractures of femurs and radius by combining various detection methods. Neck Shaft Angle (NSA), Gabor Orientation (GO), Markov Random Field (MRF), and Intensity Gradient Direction (IGD) are general methods extracted by these methods. Bayesian classifier is applied with above features. According to results obtained individual classifier gives low fracture detection rate as well as low classification accuracy.

A method is presented to detect femur fractures by texture analysis of trabeculae. For this a set of healthy and fracture training samples are each modeled by a multivariate Gaussian function. According to performed experiments and results Bayesian has no false alarm but it is less effective in detecting fractures. [12]

\subsubsection{Gini-Support Vector Machine (G-SVM)}

[4] Lum, Vineta and [11] Lim, Sher Ee, et al describes a systematic method that was employed to determine the kernel function and parameter values that produce the best overall performance on the training and testing sets. Gaussian kernel was found to give better results. According to the results GiniSVM gives better results in terms of both accuracy and sensitivity.

\subsubsection{Support Vector Machine (SVM)}

[1] [2] Mahendran presents ensemble system for the purpose of detecting fractures in X-ray images. Total 11 texture features used are GLCM features namely Contrast, Homogeneity, Energy, Entropy, Mean, Variance, Standard Deviation. Apart from this Gabor Orientation (GO), Markov Random Field (MRF), and Intensity Gradient Direction (IGD) features. Hold-out method is used for Partitioning dataset and Majority voting scheme is used for Aggregation of obtained results followed with SVM classifier. To implement the principles of SVMs, the LIB-SVM is used. Feature values which are linearly scaled are taken and linear kernel was used.

[11] Lim, Sher Ee, et al describes an approach in detecting fractures of femurs and radius by combining various detection methods. Neck Shaft Angle (NSA), Gabor Orientation (GO), Markov Random Field (MRF), and Intensity Gradient 
Direction (IGD) are general methods extracted by these methods. For the method verification Radial Basis Function (RBF) is chosen as kernel function. According to empirical results they have proved that SVM classifies more accurately. According to test results SVM has better fracture detection rate, classification accuracy and low false alarm rate.

A method is presented to detect femur fractures by texture analysis of trabeculae. For this a set of healthy and fracture training samples are each modeled by a multivariate Gaussian function. According to the experimental test results SVM has highest classification accuracy and fracture detection rate. [12]

[13] He, Joshua, Wee Leow, and Tet Howe. This paper presented a new divide-and-conquer approach for fracture detection by partitioning the problem into smaller subproblems in SVM's kernel space, and training an SVM to specialize in solving each sub-problem.

\subsubsection{Hierarchy of SVM (H-SVM)}

[13] He, Joshua, Wee Leow, and Tet Howe presented a new divide-and-conquer approach for fracture detection by partitioning the problem into smaller sub-problems in SVM's kernel space, and training an SVM to specialize in solving each sub-problem. Hierarchical SVM's namely SVM + , HSVM, and H-SVM- are used along with Intensity Gradient (IG) and Gabor Orientation (GO). According to test results and performance comparison SVM+ is better than others. Also, H-SVM uses sets optimally to achieve high performance. This is followed with the results that show with feature combination, H-SVM- achieves higher accuracy and same sensitivity as of SVM, SVM+, and H-SVM. The hierarchy of SVM's performs better than an individual SVM solving the whole problem.

\subsubsection{Nä̈ve Bayes (NB)}

[1] [2] Mahendran presents ensemble system for the purpose of detecting fractures in X-ray images. Total 11 texture features used are GLCM features namely Contrast, Homogeneity, Energy, Entropy, Mean, Variance, Standard Deviation. Apart from this Gabor Orientation (GO), Markov Random Field (MRF), and Intensity Gradient Direction (IGD) features. Hold-out method is used for Partitioning dataset and Majority voting scheme is used for Aggregation of obtained results. Considering experimental results Naive Bayes (NB) has comparatively low fracture detection rate and classification accuracy and high false alarm rate.

\subsection{Hybrid Classifiers}

\subsubsection{BPNN \& SVM}

[1] [2] Mahendran presents ensemble system for the purpose of detecting fractures in X-ray images. Total 11 texture features used are GLCM features namely Contrast, Homogeneity, Energy, Entropy, Mean, Variance, Standard Deviation. Apart from this Gabor Orientation (GO), Markov Random Field (MRF), and Intensity Gradient Direction (IGD) features. Hold-out method is used for Partitioning dataset and Majority voting scheme is used for Aggregation of obtained results. BPNN with the tangent-sigmoid transfer function and LIB-SVM are used but following the experimental results individual classifiers has low performance metrics that that of combined classifiers. The combination of BPNN and SVM produces high quality results in terms of fracture detection rate and classification accuracy. Also the time efficiency calculations show that they have better performance.

\subsubsection{BPNN \& NB}

[1] [2] Mahendran presents ensemble system for the purpose of detecting fractures in X-ray images. Total 11 texture features used are GLCM features namely Contrast, Homogeneity, Energy, Entropy, Mean, Variance, Standard Deviation. Apart from this Gabor Orientation (GO), Markov Random Field (MRF), and Intensity Gradient Direction (IGD) features. Hold-out method is used for Partitioning dataset and Majority voting scheme is used for Aggregation of obtained results. Following the experimental results it is clear that combination of BPNN and NB produces results with comparatively low results in terms of fracture detection rate and classification accuracy. Also they take longer training and testing time.

\subsubsection{SVM \& $N B$}

[1] [2] Mahendran presents ensemble system for the purpose of detecting fractures in X-ray images. Total 11 texture features used are GLCM features namely Contrast, Homogeneity, Energy, Entropy, Mean, Variance, Standard Deviation. Apart from this Gabor Orientation (GO), Markov Random Field (MRF), and Intensity Gradient Direction (IGD) features. Hold-out method is used for Partitioning dataset and Majority voting scheme is used for Aggregation of obtained results. Experimental results approach defines that SVM and NB combination produces high quality results.

\subsubsection{BPNN, SVM \& NB}

[1] [2] Mahendran presents ensemble system for the purpose of detecting fractures in X-ray images. Total 11 texture features used are GLCM features namely Contrast, Homogeneity, Energy, Entropy, Mean, Variance, Standard Deviation. Apart from this Gabor Orientation (GO), Markov Random Field (MRF), and Intensity Gradient Direction (IGD) features. Hold-out method is used for Partitioning dataset and Majority voting scheme is used for Aggregation of obtained results. Here when the 3 classifiers namely BPNN, SVM, and NB are used as a system shows degraded performance. Also the speed of execution is longer.

\subsubsection{BAYESIAN \& SVM}

A method is presented to detect femur fractures by texture analysis of trabeculae. For this a set of healthy and fracture training samples are each modeled by a multivariate Gaussian function. Approach in terms of performance metrics Bayesian and SVM improve both classification accuracy and fracture detection rate. [12]

\subsubsection{BAYESIAN \& NSA}

A method is presented to detect femur fractures by texture analysis of trabeculae. For this a set of healthy and fracture training samples are each modeled by a multivariate Gaussian function. According to concluded statement combination of NSA and Bayesian achieves the best overall performance in terms of high classification accuracy and fracture detection rate. [12]

\subsubsection{SVM \& NSA}

A method is presented to detect femur fractures by texture analysis of trabeculae. For this a set of healthy and fracture training samples are each modeled by a multivariate Gaussian function. Following the performance calculations provided in paper NSA and SVM has comparatively low performance. [12]

\section{DISCUSSION}

Bone fracture is common problem and even in most developed countries the numbers of fractures are increasing 
rapidly. So the fully automatic detection and classification of fractures is an important but difficult problem.

Among the fracture detection techniques discussed, fracture detection using classifiers in X-ray image appears promising. However, there is a need to accurately detect fracture using minimum and computationally less expensive feature and classifiers.

According to survey of different papers it is noted that the fracture detection rate of individual classifier is not very high, they can complement each other in fracture detection. So by combining individual classifiers both fracture detection rate and classification accuracy are improved.

Classifiers discussed above have been applied with combination of one or two among different feature parameters. Between above discussed classifiers, it is noted that the performance of SVM and Bayesian gives better performance in terms of fracture detection rate, classification accuracy.

One of the most important facts is minimum and computationally less expensive feature and classifiers should be used.

As per the authors [1] [2] [11] [12] the performance metrics are shown in figure $1 \& 2$ in chart form to help the reader to understand and analyze.

The performance measures are:

- Fracture Detection Rate:

The number of correctly detected fractured samples over the number of fractured samples.

- False Alarm Rate:

The number of wrongly classified healthy samples over the number of healthy samples.

- Classification Accuracy:

The number of correctly classified samples over the total number of samples.

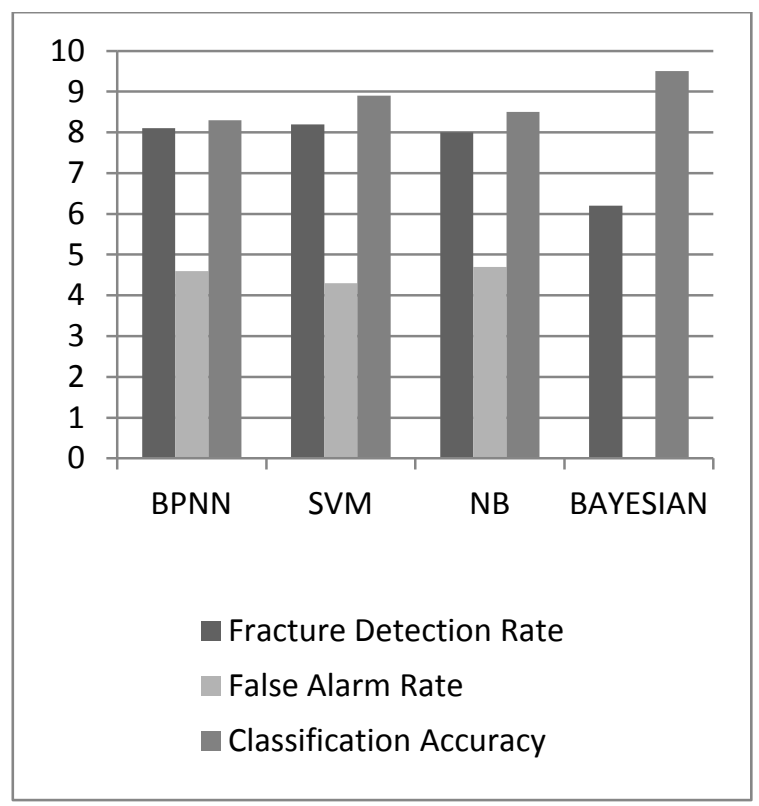

Figure 1: Performance of Individual Classifier

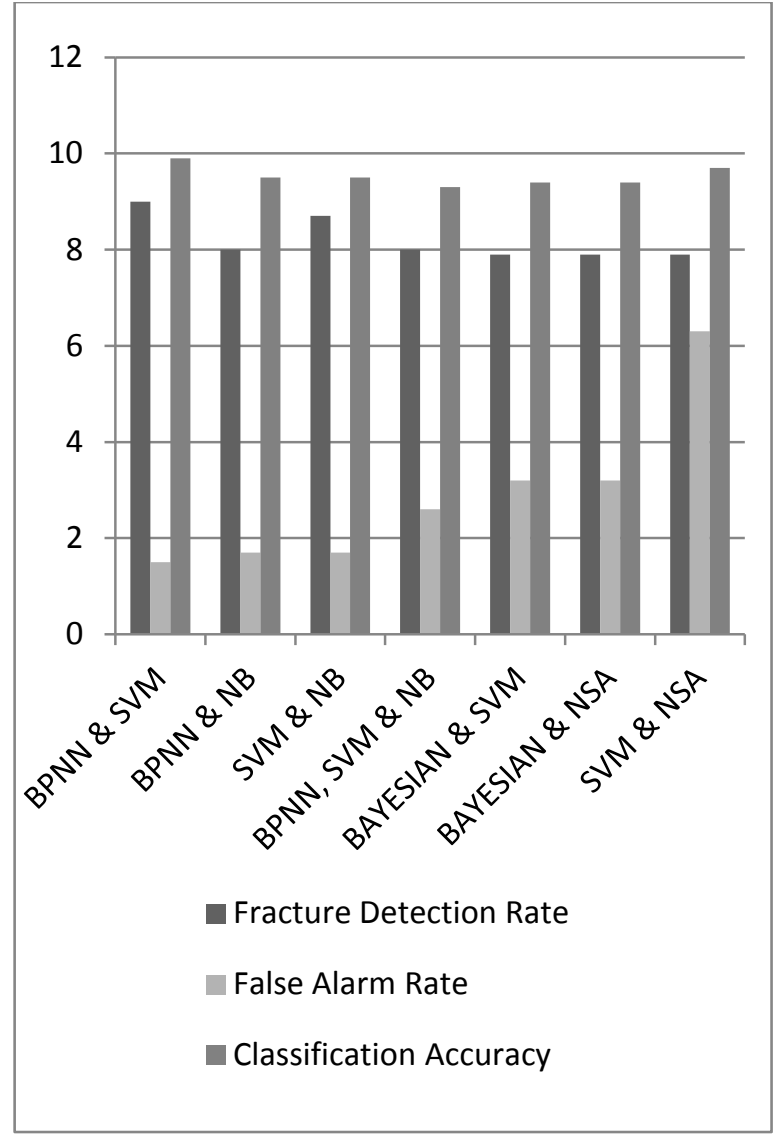

Figure 2: Performance of Hybrid Classifiers

\section{REFERENCES}

[1] Mahendran, S. K., and S. Santhosh Baboo. "Ensemble Systems for Automatic Fracture Detection." IACSIT International Journal of Engineering and Technology, Vol. 4, No. 1, February 2012.

[2] Mahendran, S. K., and S. Santhosh Baboo. "An Enhanced Tibia Fracture Detection Tool Using Image Processing and Classification Fusion Techniques in XRay Images"(2011).

[3] Nathanael E Jacob and M. V. Wyawahare. "Survey of Bone Fracture Detection Techniques". International Journal of Computer Applications 71.17(2013):31-34.

[4] Lum, Vineta Lai Fun, et al. "Combining classifiers for bone fracture detection in X-ray images." Image Processing, 2005. ICIP 2005. IEEE International Conference on. Vol. 1. IEEE, 2005.

[5] Jia,Y.,and Y.Jiang. "Active contour model with shape constraints for bone fracture detection." Computer Graphics, Imaging and Visualisation, 2006 International Conference on. IEEE, 2006.

[6] Liang, Jian, et al. "Fracture identification of X-ray image." Wavelet Analysis and Pattern Recognition (ICWAPR), 2010 International Conference on. IEEE, 2010.

[7] Donnelley, Martin William. "Computer aided long-bone segmentation and fracture detection." Diss. Flinders University, Faculty of Science and Engineering. 2008. 
[8] Wei, Zheng, and Zhang Liming. "Study on recognition of the fracture injure site based on X-ray images." Image and Signal Processing (CISP), 2010 3rd International Congress on. Vol. 4. IEEE, 2010.

[9] Smith, Rebecca, et al. "Detection of fracture and quantitative assessment of displacement measures in pelvic X-RAY images." Acoustics Speech and Signal Processing (ICASSP), 2010 IEEE International Conference on. IEEE, 2010

[10] Linda, C. Harriet, and G. Wiselin Jiji. "Crack detection in X-ray images using fuzzy index measure." Applied Soft Computing 11.4 (2011): 3571-3579.

[11] Lim, Sher Ee, et al. "Detection of femur and radius fractures in x-ray images. "Proc. 2nd Int. Conf. on Advances in Medical Signal and Info. Proc. 2004.

[12] Yap, Dennis Wen- Hsiang, et al. "Detecting femur fractures by texture analysis of trabeculae." Pattern Recognition, 2004. ICPR2004. Proceedings of the 17th International Conference on. Vol. 3. IEEE, 2004.

[13] He, Joshua, Wee Leow, and Tet Howe. "Hierarchical classifiers for detection of fractures in x-ray images." Computer Analysis of Images and Patterns. Springer Berlin/Heidelberg, 2007.
[14] Giger, Maryellen L., Nico Karssemeijer, and Samuel G. Armato III. "Computer-aided diagnosis in medical imaging." IEEE Trans. Med. Imaging 20.12 (2001): $1205-1208$

[15] Umadevi, N., and S. N. Geethalakshmi. "Enhanced Segmentation Method for bone structure and diaphysis extraction from x-ray images." International Journal of Computer Applications. 37.3 (2012): 30-36.

[16] Berbaum, Kevin S., et al. "Gaze dwell times on acute trauma injuries missed because of satisfaction of search.' Academic radiology 8.4 (2001): 304-314.

[17] Syiam, Mostafa, Mostafa Abd El-Aziem, and Mohamed El- Menshawy. "AdAgen: Adaptive Interface Agent for X-Ray Fracture Detection." International Journal of Computing \& Information Sciences 2.3 (2004).

[18] Peng, Tian Tai. "Detection of Femur Fractures in X-ray images." Master of Science Thesis, National University of Singapore (2002).

[19] Tian, Tai, et al. "Computing neck-shaft angle of femur for x-ray fracture detection." Computer Analysis of Images and Patterns. Springer Berlin/Heidelberg, 2003.

[20] Chai, Hum Yan, et al. "Gray-level co-occurrence matrix bone fracture detection." American Journal of Applied Sciences 8.1 (2011): 26-32. 